\title{
Blind Multiuser Equalization for Time-Varying Channels
}

\author{
H. Artés and F. Hlawatsch ${ }^{1}$ \\ Institute of Communications and Radio-Frequency Engineering \\ Vienna University of Technology \\ Gusshausstrasse 25/389, A-1040 Vienna, Austria (Europe) \\ email: hartes@aurora.nt.tuwien.ac.at \\ web: http://ww. nt.tuwien.ac.at/dspgroup/time.html
}

\begin{abstract}
We propose two methods for deterministic blind equalization of linear time-varying (LTV) channels with multiple users. Our approach is based on an LTI multichannel representation of LTV channels. With the first method, the multiuser channel is equalized up to an instantaneous mixture that is subsequently resolved by means of a POCS (projections onto convex sets) algorithm. The second method performs the entire equalization (including resolution of the instantaneous mixture) by means of a POCS algorithm. Simulation results demonstrate the good performance of our methods.
\end{abstract}

\section{INTRODUCTION}

Two POCS (projections onto convex sets) algorithms for deterministic blind equalization of linear time-varying (LTV) channels have recently been proposed [1]. In this paper, these algorithms are extended to the multiuser case. We consider $D$ users that transmit signals $s^{(d)}[n](d=1,2, \cdots, D)$ over discretetime LTV channels. The receiver employs multiple antennas or interpolation to observe $M$ signals

$$
x^{(i)}[n]=\sum_{d=1}^{D} \sum_{m=0}^{L-1} h^{(i, d)}[n, m] s^{(d)}[n-m], \quad i=1,2, \cdots, M,
$$

where $n=0,1, \cdots, N-1$ (i.e., the output signals $x^{(i)}[n]$ are observed over the interval $[0, N-1]), h^{(i, d)}[n, m]$ denotes the impulse response of the LTV channel that maps $s^{(d)}[n]$ into $x^{(i)}[n]$, and $L-1$ is the channel's maximum time delay. In a communications application, the input signals $s^{(d)}[n]$ will typically be sequences of data symbols (the transmit and receive filters are incorporated in the channel [2]).

The paper is organized as follows. An LTI multichannel representation of the multiuser LTV channel is discussed in Section II. In Section III, the overall framework for the blind equalization method is explained. Following [2, 3], we show how the LTV multiuser channel can be equalized up to an instantaneous mixture. Section IV presents a POCS algorithm for resolving this instantaneous mixture. Section $V$ presents an improved equalization method that is entirely based on a POCS algorithm. Finally, simulation results are provided in Section VI.

\section{LTi Multichannel Representation}

The input-output relation (1) can be rewritten as (cf. [1])

$$
x^{(i)}[n]=\sum_{d=1}^{D} \sum_{l=0}^{N_{D}-1} \sum_{m=0}^{L-1} h_{l}^{(i, d)}[m] s_{l}^{(d)}[n-m]
$$

where $h_{l}^{(i, d)}[m] \triangleq S_{h}^{(i, d)}[m, l] e^{j 2 \pi \frac{l m}{N}}$ with the (delay-Doppler) spreading function [4] $S_{h}^{(i, d)}[m, l]=\sum_{n=0}^{N-1} h^{(i, d)}[n, m] e^{-j 2 \pi \frac{d n}{N}}$,

\footnotetext{
${ }^{1}$ Funding by FWF grant P12228-TEC.
}

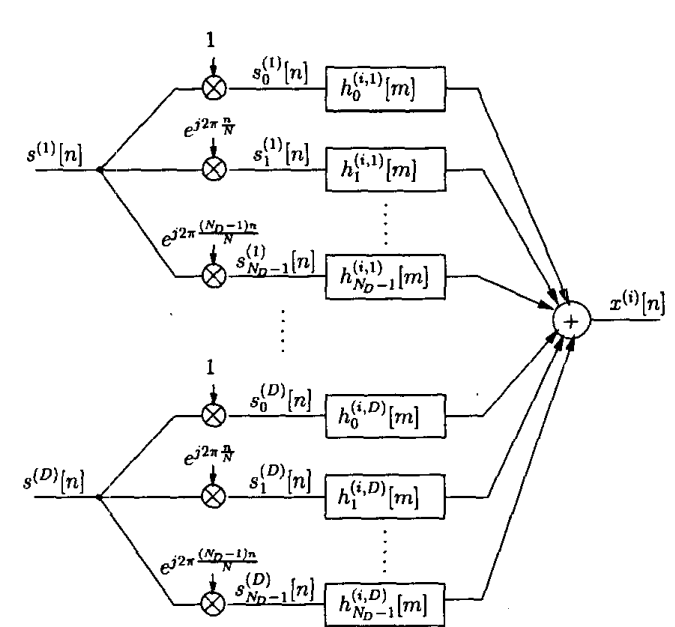

Fig. 1: LTI multichannel representation of a multiuser LTV channel.

$s_{l}^{(d)}[n] \triangleq s^{(d)}[n] e^{j 2 \pi \frac{l n}{N}}$, and $N_{D}$ is the maximum Doppler shift. This corresponds to an LTI multichannel representation of each individual LTV channel, where each subchannel consists of a modulator (Doppler shift) and an LTI filter (see Fig. 1). Practically arbitrary channels-including channels with diffuse scattering-are characterized by a finite set of LTI filters associated with uniformly spaced, discrete Doppler shifts. Equivalently, $h^{(i, d)}[n, m]$ is expanded (cf. [5]) into the basis functions $e^{j 2 \pi \frac{n}{N}}$ that naturally arise through discretization of a continuous-time channel. Estimation of basis functions or Doppler shifts is not required. Note, however, that all algorithms presented here can also be used with channel models that assume nonuniformly spaced Doppler shifts (e.g. [5]).

In what follows, we assume that for the $d$ th user, $K_{d}$ of the $N_{D}$ subchannels in Fig. 1-corresponding to specific Doppler shifts $l_{k}^{(d)} \in\left[0, N_{D}-1\right]$ with $k=1,2, \cdots, K_{d}$ and $d=1,2, \cdots, D-$ are active. That is, only the subchannel impulse responses $h_{l}^{(i, d)}[m]$ with $l=l_{k}^{(d)}$ are nonzero. (This is no restriction since we allow $K_{d}=N_{D}$.) Thus, (2) becomes

$$
x^{(i)}[n]=\sum_{d=1}^{D} \sum_{k=1}^{K_{d}} \sum_{m=0}^{L-1} h_{k}^{(i, d)}[m] s_{k}^{(d)}[n-m]
$$

where (with an abuse of notation) we have written $h_{k}^{(i, d)}[m] \triangleq$ $h_{l_{k}^{(d)}}^{(i, d)}[m]$ and $s_{k}^{(d)}[n] \triangleq s_{l_{k}^{(d)}(n)}^{(d)}[n]$ for notational simplicity. The model parameters that will be relevant to our method are the "active" Doppler shifts $l_{k}^{(d)}$ and the length $L$ of the corresponding channel impulse responses $h_{k}^{(i, d)}[m]$ (all $h_{k}^{(i, d)}[m]$ are assumed to have the same length). For WSSUS channels, these 
model parameters can be deduced from the scattering function $[4,6]$ corresponding to the respective user (we assume that the $M$ LTV channels corresponding to a given user possess the same scattering function). Because the scattering function does not change with time, it is much easier to estimate than the channel itself $[7,8]$. In the following, the active Doppler frequencies $l_{k}^{(d)}$ of each user will be arranged in strictly increasing order, i.e., $l_{k}^{(d)}>l_{k^{\prime}}^{(d)}$ for $k>k^{\prime}$. We will call the sequence of positive numbers $P_{d} \triangleq\left(l_{2}^{(d)}-l_{1}^{(d)}, l_{3}^{(d)}-l_{2}^{(d)}, \cdots, l_{K_{d}}^{(d)}-l_{K_{d}-1}^{(d)}\right)$ the Doppler profile of the $d$ th user.

\section{Blind Multiuser Equalization}

The LTV multiuser channel representation in Fig. 1 is closely related to multiuser communication over LTI channels. There are however two differences: we have $K=\sum_{d=1}^{D} K_{d}$ (rather than $D$ ) channels per antenna, and all subchannel input signals associated with a specific user are related as

$$
s_{k}^{(d)}[n]=s^{(d)}[n] e^{j 2 \pi \sum_{h}^{(d)} n}
$$

This analogy to the LTI case allows to equalize the LTV multiuser channel up to an instantaneous mixture $[2,3]$. We first reformulate (3) analogously to $[2,3]$. Let

$$
\mathbf{H}_{d}[m] \triangleq\left[\begin{array}{ccc}
h_{1}^{(1, d)}[m] & \cdots & h_{K_{d}}^{(1, d)}[m] \\
\vdots & & \vdots \\
h_{1}^{(M, d)}[m] & \cdots & h_{K_{d}}^{(M, d)}[m]
\end{array}\right]
$$

$\mathbf{H}[m] \triangleq\left[\mathbf{H}_{1}[m] \cdots \mathbf{H}_{D}[m]\right]$, and $\mathbf{H} \triangleq[\mathbf{H}[0] \cdots \mathbf{H}[L-1]]$, and define the following channel matrix of size $M u \times K(L+u-1)$ in which $H$ is stacked $u$ times with shifts to the left by $K$ positions each (the parameter $u$ is sometimes called smoothing factor [2]),

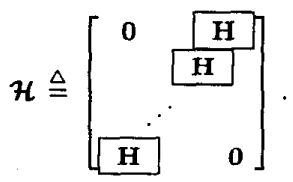

Next, we define a vector of the modulated input samples of all users, $\mathbf{s}[n] \triangleq\left[\mathbf{s}_{1}^{T}[n] \cdots \mathbf{s}_{D}^{T}[n]\right]^{T}$ with $\mathbf{s}_{d}[n] \triangleq$ $\left[s_{1}^{(d)}[n] \cdots s_{K_{d}}^{(d)}[n]\right]^{T}$, and we form the following block-Toeplitz input matrix of size $K(L+u-1) \times(N-u+1)$,

$$
\mathcal{S} \triangleq\left[\begin{array}{cccc}
\mathbf{s}[u-1] & \mathbf{s}[u] & \cdots & \mathbf{s}[N-1] \\
\mathbf{s}[u-2] & \mathbf{s}[u-1] & \cdots & \mathbf{s}[N-2] \\
\vdots & \vdots & \ddots & \vdots \\
\mathbf{s}[-L+1] & \mathbf{s}[-L+2] & \cdots & \mathbf{s}[N-L-u+1]
\end{array}\right] .
$$

Finally, we define the output vector $\mathbf{x}[n] \triangleq\left[x^{(1)}[n] \cdots x^{(M)}[n]\right]^{T}$ and form the following block-Hankel output matrix of size $M u \times$ $(N-u+1)$

$$
\boldsymbol{X} \triangleq\left[\begin{array}{ccccc}
\mathbf{x}[0] & \mathbf{x}[1] & \cdots & \mathbf{x}[N-u-1] & \mathbf{x}[N-u] \\
\mathbf{x}[1] & \mathbf{x}[2] & \cdots & \mathbf{x}[N-u] & \mathbf{x}[N-u+1] \\
\vdots & \vdots & . & \vdots & \vdots \\
\mathbf{x}[u-1] & \mathbf{x}[u] & \cdots & \mathbf{x}[N-2] & \mathbf{x}[N-1]
\end{array}\right]
$$

Now (3) can be written as the matrix relation (cf. $[2,3]$ )

$$
\boldsymbol{X}=\mathcal{H} \mathcal{S}
$$

Blind equalization corresponds to calculation of the input/ symbol matrix $\mathcal{S}$ from the known (received) matrix $\mathcal{X}$. For this to be possible, $\mathcal{S}$ must be a wide matrix and the row span of $\boldsymbol{X}$ must be equal to the row span of $\mathcal{S}[2,3]$. This, in turn, requires that $\mathcal{H}$ is a square or tall matrix and has full rank. These requirements lead to the necessary conditions [2] $u \geq \frac{K(L-1)}{M-K}$, with $M>K$, and $N>K L+(K+1)(u-1)$.

The input matrix $\mathcal{S}$ in (5) is a block-Toeplitz matrix that is generated by the vectors $\mathbf{s}[-L+1], \mathbf{s}[-L+2], \cdots, \mathbf{s}[N-1]$ or, equivalently, by the "generating matrix"

$$
\mathbf{s} \triangleq[\mathrm{s}[-L+1] \mathbf{s}[-L+2] \cdots \mathrm{s}[N-1]]
$$

of size $K \times(N+L-1)$. Thus, our equalization problem can be equivalently phrased as calculation of $S$ from $\mathcal{X}$. This can be done as follows:

Step 1: Using a singular value decomposition (SVD), the row span of $\mathcal{S}$ is calculated from $\boldsymbol{X}$ [2].

Step 2: Another SVD is used to construct a $K(L+u-1) \times$ $(N-u+1)$ block-Toeplitz matrix $\mathcal{S}_{A}$ whose row span equals that of $\mathcal{S}[2,3]$. It can be shown that the $K \times(N+L-1)$ generating matrix of $\mathcal{S}_{A}$ can be written as $S_{A}=A S$, where $A$ is an unknown invertible matrix of size $K \times K$ [2]. Due to the SVD construction, the rows of $\mathbf{S}_{A}$ are orthonormal.

At this point, $S$ is determined up to an ambiguity corresponding to the unknown mixing matrix $\mathbf{A}$, which mixes up both the modulated versions of the input signal of one given user and the signals belonging to different users. Hence, the dispersive LTV multiuser channel has been reduced to an instantaneous mixture.

Step 3: This instantaneous mixture is resolved whereby $\mathbf{S}$ and, in turn, all the users' scalar input sequences $s^{(d)}[-L+$ $1], s^{(d)}[-L+2], \cdots, s^{(d)}[N-1]$ for $d=1 \cdots D$ are obtained up to constant factors. Thus, we have achieved both multiuser separation and equalization of the dispersive LTV multiuser channel.

\section{Resolving the Instantaneous Mixture}

We now present a POCS method for resolving the instantaneous mixture as required in Step 3. This method exploits the modulation relations (4) between the $s_{k}^{(d)}[n]$. Allowing for time offsets $n_{d}$ which may occur in practice, these relations are $s_{k}^{(d)}[n]=s^{(d)}[n] e^{j 2 \pi l_{k}^{(d)}\left(n_{d}+n\right) / N}$ on, formulated in terms of $\mathrm{S}$,

$\mathbf{S}=\mathbf{M}\left(n_{1}, \cdots, n_{D}\right) \mathbf{D}=\left[\left(\mathbf{M}_{1}\left(n_{1}\right) \mathbf{D}_{1}\right)^{T} \cdots\left(\mathbf{M}_{D}\left(n_{D}\right) \mathbf{D}_{D}\right)^{T}\right]^{T}$.

Here, we have defined the $K \times D(N+L-1)$ block diagonal "modulation matrix" $\mathbf{M}\left(n_{1}, \cdots, n_{D}\right) \triangleq \operatorname{diag}\left\{\mathbf{M}_{1}\left(n_{1}\right), \cdots, \mathbf{M}_{D}\left(n_{D}\right)\right\}$, where

$$
\mathbf{M}_{d}\left(n_{d}\right) \triangleq\left[\begin{array}{cccc}
W_{N}^{-n_{d} l_{1}^{(d)}} & W_{N}^{-\left(n_{d}+1\right) l_{1}^{(d)}} & \cdots & W_{N}^{-\left(n_{d}+N+L-1\right) l_{1}^{(d)}} \\
\vdots & \vdots & & \vdots \\
W_{N}^{-n_{d} l_{K_{d}}^{(d)}} & W_{N}^{-\left(n_{d}+1\right) l_{K_{d}}^{(d)}} & \cdots & W_{N}^{-\left(n_{d}+N+L-1\right) l_{K_{d}}^{(d)}}
\end{array}\right]
$$

with $W_{N}^{k} \triangleq e^{-j 2 \pi \frac{k}{N}}$, and the $D(N+L-1) \times(N+L-1)$ input matrix $\mathbf{D} \triangleq\left[\mathrm{D}_{1}^{T} \cdots \mathrm{D}_{D}^{T}\right]^{T}$ with $\mathbf{D}_{d} \triangleq \operatorname{diag}\left\{s^{(d)}[-L+\right.$ 1], $\left.s^{(d)}[-L+2], \cdots, s^{(d)}[N-1]\right\}$.

Given a matrix $\mathbf{S}_{A}$ and a modulation matrix $\mathbf{M}$ (hereafter, $\mathbf{M}$ and $\mathbf{M}_{d}$ are short for $\mathbf{M}\left(n_{1}, \cdots, n_{D}\right)$ and $\mathbf{M}_{d}\left(n_{d}\right)$, respectively), the desired generating matrix $\mathbf{S}$ satisfies the following two structural properties:

1. $\mathbf{S}$ has. modulation structure, i.e., $\mathbf{S}=\mathbf{M D}=$ $\left[\left(\mathbf{M}_{1} \mathbf{D}_{1}\right)^{T} \ldots\left(\mathbf{M}_{D} \mathbf{D}_{D}\right)^{T}\right]^{T}$ with all $\mathbf{D}_{d}$ diagonal;

2. the row span of $\mathbf{S}$ equals the row span of $\mathbf{S}_{A}$ (since $\mathbf{S}_{A}=$ AS with A nonsingular). 
It follows that $\mathbf{S} \in \mathcal{A} \cap \mathcal{B}$ where $\mathcal{A}$ denotes the linear subspace of all matrices $\left[\left(\mathbf{M}_{1} \mathbf{D}_{1}\right)^{T} \cdots\left(\mathbf{M}_{D} \mathbf{D}_{D}\right)^{T}\right]^{T}$ with $\mathbf{M}_{d}$ given and $\mathbf{D}_{d}$ diagonal, and $\mathcal{B}$ denotes the linear subspace of all matrices whose row span lies in the row $\operatorname{span}$ of $\mathbf{S}_{A}$, i.e., of all matrices of the form $\mathrm{BS}_{A}$ with some $K \times K$ matrix $\mathrm{B}$.

Since both $\mathcal{A}$ and $\mathcal{B}$ are linear subspaces and thus convex, the formulation $S \in \mathcal{A} \cap \mathcal{B}$ suggests a POCS algorithm [9] for instantaneous mixture resolution. This algorithm is iterative and consists in alternately projecting the iterated version of the matrix $\mathbf{S}$ onto $\mathcal{A}$ and $\mathcal{B}$.

Projection onto $\mathcal{A}$ : The projection onto $\mathcal{A}$ amounts to forming $\mathbf{S}^{(i)}=\mathbf{M D}^{(i)}=\left[\left(\mathbf{M}_{1} \mathbf{D}_{1}^{(i)}\right)^{T} \ldots\left(\mathbf{M}_{D} \mathbf{D}_{D}^{(i)}\right)^{T}\right]^{T}$, where the nonzero (diagonal) elements of $D_{d}^{(i)}$ can be shown to be given by

$$
\left(\mathbf{D}_{d}^{(i)}\right)_{n, n}=\frac{1}{K_{d}} \sum_{k=1}^{K_{d}}\left(\mathbf{S}^{(i-1)}\right)_{k+k_{d}, n}\left(\mathbf{M}_{d}\right)_{k, n}^{*}
$$

Here, $k_{d}=\sum_{j=1}^{d-1} K_{j}$ and $\mathbf{S}^{(i-1)}$ is the result of the previous iteration (i.e., the projection onto $\mathcal{B}$ ).

Projection onto $\mathcal{B}$ : The projection onto $\mathcal{B}$ amounts to forming $\mathbf{S}^{(i)}=\mathbf{B}^{(i)} \mathbf{S}_{A}$, where it can be shown that

$$
\mathbf{B}^{(i)}=\mathbf{S}^{(i-1)} \mathbf{S}_{A}^{\#} \text {. }
$$

Here, $\mathbf{S}^{(i-1)}$ is the result of the previous iteration (i.e., the projection onto $\mathcal{A}$ as explained above) and $\mathbf{S}_{A}^{\#}$ is the pseudo-inverse of $\mathbf{S}_{A}$. Since $\mathbf{S}_{A}$ is a wide matrix with orthonormal rows, there is simply $\mathbf{S}_{A}^{\#}=\mathbf{S}_{A}^{H}$.

The POCS algorithm is guaranteed to converge to an intersection point, i.e., $\mathbf{s}^{(\infty)} \in \mathcal{A} \cap \mathcal{B}$ [9]. In our experiments, assuming that different users have different Doppler profiles $\left(P_{d} \neq P_{d^{\prime}}\right.$ for $\left.d \neq d^{\prime}\right)$, we always observed that $\mathbf{S}^{(\infty)}$ yielded the desired user signals $s^{(d)}[-L+1], s^{(d)}[-L+2], \cdots, s^{(d)}[N-1]$ for all $d$ up to constant complex factors $c_{d}$. That is, we obtained $\mathbf{S}^{(\infty)}=\mathbf{C S}$ where $\mathbf{S}$ is the desired (true) generating matrix and $\mathbf{C}=\operatorname{diag}\left\{\mathbf{C}_{1} \cdots \mathbf{C}_{D}\right\}$ with the diagonal $K_{d} \times K_{d}$ matrice $\mathbf{C}_{d}=c_{d} \mathbf{I}$ whose diagonal elements are the factors $c_{d}$ (as is usual for blind estimation, the signal of each user can only be recovered up to an unknown constant factor). Note that, upon convergence, the user signals $s^{(d)}[-L+1], s^{(d)}[-L+2], \cdots, s^{(d)}[N-1]$ (multiplied by $c_{d}$ ) are easily obtained from the diagonal signa matrices $D_{d}^{(\infty)}$ in (6). We also observed that the algorithm does not require knowledge of the correct time offsets $n_{d}$; in fact, arbitrary values of $n_{d}$ (e.g., $n_{d} \equiv 0$ ) can be used. A theoretical proof of these facts is currently under investigation (see [1] for a proof in the single-user case $D=1$ ).

The condition $P_{d} \neq P_{d^{\prime}}$ for $d \neq d^{\prime}$ can be shown to be necessary for user separation. If some users have identical Doppler profiles, the algorithm yields an instantaneous mixture of these users (which can still be resolved using a finite alphabet property, see e.g. [2]) aithough it correctly separates the other users.

The convergence speed of the POCS algorithm depends on the initialization, $\mathbf{S}^{(0)}$. In the semiblind case, we could use some known symbols to calculate a good initialization. However, our simulations showed that initialization is not very critical since the convergence of the POCS algorithm always was much faster than the two SVDs required to obtain $S_{A}$ from $\mathcal{X}$. The convergence speed can be increased by relaxation [9] and/or by using knowledge of the symbol alphabet. The latter approach, however, introduces a nonconvex set and thus convergence to the desired solution is no longer guaranteed.

\section{POCS Multiúser Equalization Method}

A computationally intensive part of the three-step method of Section III is the SVD in Step 2 that is used to construct the generating matrix $\mathbf{S}_{A}$. This SVD can be avoided by noting that according to Sections III and IV the matrix $\mathcal{S}$ satisfies the following two structural properties:

1. $\mathcal{S}$ is block Toeplitz and its generating matrix has modulation structure, i.e., $\mathbf{S}=\mathbf{M D}=$ $\left[\left(\mathrm{M}_{1} \mathbf{D}_{1}\right)^{T} \ldots\left(\mathbf{M}_{D} \mathbf{D}_{D}\right)^{T}\right]^{T}$ with all $\mathbf{D}_{d}$ diagonal;

2. the row span of $\mathcal{S}$ equals the row span of $\boldsymbol{X}$ (since $\boldsymbol{X}=$ $\mathcal{H} \mathcal{S}$ where $\mathcal{H}$ is square or tall with full rank)

It follows that $\mathcal{S} \in \mathcal{A} \cap \mathcal{B}$ where $\mathcal{A}$ denotes the linear subspace of all block Toeplitz matrices with generating matrix $\left[\left(\mathbf{M}_{1} \mathbf{D}_{1}\right)^{T} \ldots\left(\mathbf{M}_{D} \mathbf{D}_{D}^{\prime}\right)^{T}\right]^{T}$ with $\mathbf{M}_{d}$ given and $\mathbf{D}_{d}$ diagonal, and $\mathcal{B}$ denotes the linear subspace of all matrices whose row span lies in the row span of $\boldsymbol{X}$, i.e., of all matrices of the form $\boldsymbol{B} \mathcal{X}$ with some $K(L+u-1) \times M u$ matrix $\boldsymbol{B}$. This formulation again suggests a POCS algorithm for calculating $\mathcal{S}$ that consists in alternately projecting the iterated version of the mat:rix $\mathcal{S}$ onto $\mathcal{A}$ and $\mathcal{B}$.

Projection onto $\mathcal{A}: \mathcal{S}$ being a linear structured matrix [10], it can be shown that the projection onto $\mathcal{A}$ can be performed by the following two steps:

Step 1: Enforce block Toeplitz property. Let $\mathcal{S}^{(i-1)}$ be the result of the previous iteration (projection onto $\mathcal{B}$, see be low). From $\boldsymbol{S}^{(i-1)}$, which is not block Toeplitz, we calculate a $K \times(N+L-1)$ "pseudo generating matrix" $\overrightarrow{\mathbf{S}}^{(i-1)}$ as follows. The first one of the $K$ rows of $\tilde{\mathbf{S}}^{(i-1)}$ is obtained by averaging properly aligned and zero-padded versions of the first, $(K+1)$ st $(2 K+1)$ st, etc. rows of $\mathcal{S}^{(i-1)}$. More precisely, the first row of $\mathcal{S}^{(i-1)}$ is shifted to the right by one position and added to the $(K+1)$ st row of $\mathcal{S}^{(i-1)}$, with zeros appended where necesseury. The result is again shifted to the right by one position and added to the $(2 K+1)$ st row of $\mathcal{S}^{(i-1)}$, etc. Finally the $j$ th element of the resulting row vector of length $N+L-1$ is divided by the $j$ th element of $(1,2, \cdots, K, K, \cdots, K, K-1, \cdots, 1)$ to yield the first row of $\tilde{\mathbf{S}}^{(i-1)}$. The second row of $\tilde{\mathbf{S}}^{(i-1)}$ is obtained similarly by averaging properly aligned and zero-padded versions of the second, $(K+2)$ nd, $(2 K+2)$ nd, etc. rows of $\mathcal{S}^{(i-1)}$. In this way, all $K$ rows of $\tilde{\mathbf{S}}^{(i-1)}$ are obtained.

Step 2: Enforce modulation structure. Next, we form $\mathbf{S}^{(i)}=$ $\left[\left(\mathrm{M}_{1} \mathrm{D}_{1}^{(i)}\right)^{T} \ldots\left(\mathrm{M}_{D} \mathrm{D}_{D}^{(i)}\right)^{T}\right]^{T}$, where the nonzero (diagonal) elements of $\mathbf{D}_{d}^{(i)}$ can be shown to be given by (6) with $\mathbf{S}^{(i-1)}$ replaced by $\tilde{\mathbf{S}}^{(i-1)}$. We then form the block Toeplitz matrix $\mathcal{S}^{(i)}$ generated by $\mathbf{S}^{(i)}$.

Projection onto $\mathcal{B}$ : The projection onto $\mathcal{B}$ amounts to forming $\mathcal{S}^{(i)}=\mathcal{B}^{(i)} \mathcal{X}$, where it can be shown that

$$
\boldsymbol{B}^{(i)}=\mathcal{S}^{(i-1)} \boldsymbol{X}^{\#} \text {. }
$$

Here, $\mathcal{S}^{(i-1)}$ is the result of the previous iteration (projection onto $\mathcal{A}$, see above). Note that the pseudo-inverse $\mathcal{X}^{\#}$ need only be calculated once at the beginning of the iterative procedure.

The POCS algorithm is guaranteed to converge to an intersection point, i.e., $\mathcal{S}^{(\infty)} \in \mathcal{A} \cap \mathcal{B}[9]$. With the assumption that $P_{d} \neq P_{d^{\prime}}$ for $d \neq d^{\prime}$, our experiments always yielded as generating matrix $S^{(\infty)}=C S$ where $S$ is the desired (true) generating matrix and $\mathbf{C}=\operatorname{diag}\left\{\boldsymbol{C}_{1} \cdots \mathbf{C}_{D}\right\}$ with $\boldsymbol{C}_{d}=c_{d} \mathbf{I}$ as in Section IV. Thus, the user signals $s^{(d)}[-L+1], s^{(d)}[-L+2], \cdots, s^{(d)}[N-$ 1] are again obtained up to constant complex factors $c_{d}$. 'This works even for incorrect values of the time offsets (e.g., $n_{d} \equiv 0$ can be used).

Convergence speed depends on the initialization, $\mathcal{S}^{(0)}$. In the semiblind case, some known consecutive input samples (symbols) $s^{(d)}\left[n_{1}\right], s^{(d)}\left[n_{1}+1\right], \cdots, s^{(d)}\left[n_{2}\right]$ can be used to construct a matrix $\mathcal{S}^{(0)}$ with generating matrix $\mathbf{S}^{\langle 0\rangle}=\left[\mathbf{0}_{1}\left(\mathbf{M}_{d} \mathbf{D}_{d}^{(0)}\right)^{T} \mathrm{O}_{2}\right]^{T}$ 


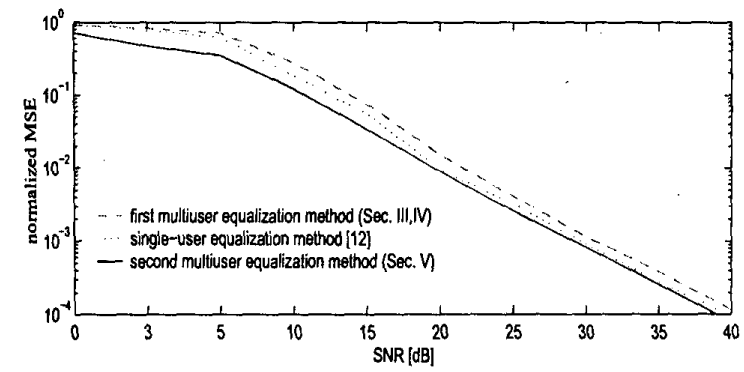

Fig. 2: MSE vs. SNR for our two blind multiuser equalization methods and the "indirect" single-user method from [11].

where $\mathbf{D}_{d}^{(0)}=\operatorname{diag}\left\{0, \cdots, 0, s^{(d)}\left[n_{1}\right], s^{(d)}\left[n_{1}+1\right], \cdots, s^{(d)}\left[n_{2}\right], 0\right.$, $\cdots, 0\}$ and $0_{1}, 0_{2}$ are zero matrices of appropriate size [12]. Convergence can again be accelerated by relaxation and/or by using knowledge of the symbol alphabet.

\section{Simulation Results}

We simulated the two multiuser equalization methods outlined in Sections III-V with $D=2$ users and $M=8$ channel output signals $x^{(i)}[n]$ (thus, $M / D=4$ ). The signals $x^{(i)}[n]$ were contaminated by white Gaussian noise and observed over an in terval of length $N=100$. The two input signals $s^{(1)}[n], s^{(2)}[n]$ were QPSK symbol sequences. Each of the $D M=16$ channels comprised $K_{d}=2$ subchannels with active Doppler shifts $l_{1}^{(1)}=0, l_{2}^{(1)}=1$ and $l_{1}^{(2)}=0, l_{2}^{(2)}=2$ and filter length $L=3$. For each simulation run, the subchannel impulse responses were generated randomly.

Fig. 2 shows the normalized mean-square errors $(\mathrm{MSE})^{1}$ obtained with our POCS methods as a function of the SNR. ${ }^{2}$ Also shown in Fig. 2 is the MSE obtained in the single-user case with the "indirect" single-user ${ }^{3}$ method presented in [11]. For the single-user method, we used the same channel parameters as above but only 4 channel output signals $x^{(i)}[n]$ since there is only one user (note that thus $M / D=4$ both in the single-user case and in the multiuser case). The MSE was estimated by averaging over 50 simulation runs. The smoothing factor was $u=10$ for all methods.

From Fig. 2, it is seen that our second multiuser method for two users (described in Section V) performs better than the single-user method for one user [11] which, in turn, performs better than our first multiuser method for two users (described in Sections III and IV).

Finally, Fig. 3 shows the estimated symbols of the two users after equalization using our second multiuser method, at an SNR of $17 \mathrm{~dB}$.

\section{CONCLUSION}

We presented two different POCS-based methods for deterministic blind equalization of linear time-varying channels with multiple users. The first method performs an initial equalization

${ }^{1}$ The normalized MSE is defined as $\sum_{d=1}^{D} \sum_{n=-L+1}^{N-1} \mid s^{(d)}[n]-$ $\left.\hat{c}_{d} \hat{s}^{(d)}[n]\right|^{2} / \sum_{d=1}^{D} \sum_{n=-L+1}^{N-1}\left|s^{(d)}[n]\right|^{2}$ averaged over all simulation runs, where $\hat{s}^{(d)}[n]$ is the estimate of $s^{(d)}[n]$ obtained with the respective method and $\hat{c}_{d}$ is the least-squares fit for the unknown factor $c_{d}$.

${ }^{2}$ The SNR is defined as $\|[\mathbf{x}[0] \cdots \mathbf{x}[N-1]]\|_{F}^{2} /\left(N M \sigma^{2}\right)$, with \|\|$_{F}$ the Frobenius norm and $\sigma^{2}$ the noise variance. The same SNR was used for each simulation run.

${ }^{3}$ Since we are not aware of any existing multiuser equalization methods for time-varying channels, we compared our methods with a single-user method.

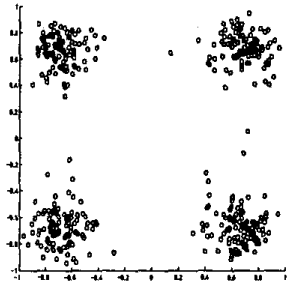

(a)

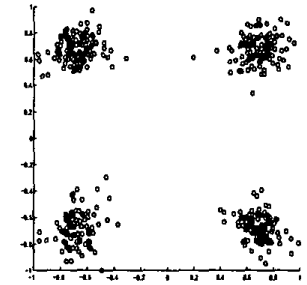

(b)
Fig. 3: Estimated symbols of the two users after equalization using our second multiuser method at an SNR of $17 \mathrm{~dB}$ : (a) user 1, (b) user 2.

according to $[2,3]$ whereby the equalization problem is reduced to an an instantaneous mixture problem; this latter problem is then resolved using a POCS algorithm. The second method uses a POCS algorithm for joint equalization and demixing, thus saving one singular value decomposition. Our simulations showed that in the presence of noise the second method performs better than the first method; it is also less computationally intensive than the first method.

\section{References}

[1] H. Artés, F. Hlawatsch, and G. Matz, "Efficient POCS algorithms for deterministic blind equalization of timevarying channels," in Proc. IEEE GLOBECOM-2000, (San Franscisco, CA), pp. 1031-1035, Nov. 2000.

[2] A. J. van der Veen, S. Talwar, and A. Paulraj, "A subspace approach to blind space-time signal processing for wireless communication systems," IEEE Trans. Signal Processing, vol. 45, pp. 173-190, Jan. 1997.

[3] H. Liu and G. Xu, "Closed form blind symbol estimation in digital communications," IEEE Trans. Signal Processing, vol. 43, pp. 2714-2723, Nov. 1995.

[4] P. A. Bello, "Characterization of randomly time-variant linear channels," IEEE Trans. Comrn. Syst., vol. 11, pp. 360-393, 1963.

[5] G. B. Giannakis and C. Tepedelenlioğla, "Basis expansion models and diversity techniques for blind identification and equalization of time-varying channels," Proc. IEEE vol. 86, pp. 1969-1986, Oct. 1998.

[6] R. S. Kennedy, Fading Dispersive Communication Channels. New York: Wiley, 1969.

[7] H. Artés, G. Matz, and F. Hlawatsch, "Unbiased scattering function estimation during data transmission," in Proc. IEEE VTC-99 Fall, (Amsterdam, The Netherlands), pp. 1535-1539, Sept. 1999.

[8] C. Tepedelenlioğlu and G. B. Giannakis, "Precoding for scattering function estimation of mobile channels using output correlations only," in Proc. 37th Allerton Conf. on Commun., Contr., Comput., Sept. 1999.

[9] P. L. Combettes, "The foundations of set theoretic estimation," Proc. IEEE, vol. 81, pp. 181-208, Feb. 1993.

[10] J. A. Cadzow, "Signal enhancement-A composite property mapping algorithm," IEEE Trans. Acoust., Speech, Signal Processing, vol. 36, pp. 49-62, Jan. 1988.

[11] H. Liu and G. B. Giannakis, "Deterministic approaches for blind equalization of time-varying channels with antenna arrays," IEEE Trans. Signal Processing, vol. 46, pp. 30033013, Nov. 1998.

[12] J. Laurila, K. Kopsa, R. Schürhuber, and E. Bonek, "Semiblind separation and detection of co-channel signals," in Proc. IEEE Int. Conf. Commun. (ICC), (Vancouver B.C. Canada), pp. 17-22, June 1999. 